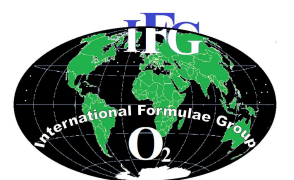

Available online at http://www.ifg-dg.org

Int. J. Biol. Chem. Sci. 10(4): 1678-1693, August 2016

International Joumnal

of Biological and

Chemical Sciences

ISSN 1997-342X (Online), ISSN 1991-8631 (Print)

Original Paper http://ajol.info/index.php/ijbcs http://indexmedicus.afro.who.int

\title{
Etude ethnobotanique des plantes alimentaires spontanées vendues dans les marchés de Yaoundé, Cameroun
}

\author{
Jean Lagarde BETTI ${ }^{1 *}$, Cédric Manga NGANKOUÉ ${ }^{1}$, Siegfried Didier DIBONG ${ }^{1}$ et \\ Alexis Eboulé SINGA ${ }^{2}$ \\ ${ }^{I}$ Département de Biologie des Organismes Végétaux, Faculté des Science, \\ Université de Douala, BP 24157 Douala, Cameroun. \\ ${ }^{2}$ Ministère des Forêts et de la Faunes (MINFOF), Cameroun. \\ *Auteur correspondant, E-mail: lagardebetti@yahoo.fr, Tel : 00 (237) 77303272
}

\section{RESUME}

Des enquêtes ethnobotaniques sur la vente des plantes alimentaires spontanées ont été réalisées de mars à avril 2012 auprès de 102 vendeurs installés dans 13 marchés de la ville de Yaoundé. Trois cent quatre citations de plantes ont été relevées faisant intervenir 29 espèces végétales dans 32 recettes alimentaires différentes. Les espèces végétales citées sont distribuées dans 25 genres et 23 familles. Les fruits ou graines sont les organes végétaux les plus cités tandis que les condiments et les amuse-gueule ou fruits de bouche constituent dans cet ordre les types d'usages les plus indiqués. Les prix de plantes varient de $0,49 \mathrm{FCFA} / \mathrm{g}$ à 20 FCFA/g pour un prix moyen du gramme de 4,28 $\pm 3,1$ FCFA ( 1 USD $=588$ FCFA). Ces prix apparaissent plus élevés comparés aux autres pays et aussi à d'autres villes du Cameroun pour les mêmes espèces végétales. Des travaux complémentaires sur le suivi des vendeurs et la collecte des données floristiques et structurales sont nécessaires pour envisager le statut de vulnérabilité de ces espèces et anticiper sur les modèles de gestion durable et plus rentable.

() 2016 International Formulae Group. All rights reserved.

Mots clés : Enquêtes ethnobotaniques, plantes alimentaires spontanées, recettes, coût, Yaoundé.

\section{Ethnobotanical study of edible wild plants sold in the Yaoundé markets, Cameroon}

\begin{abstract}
Ethnobotanical surveys were conducted among 102 sellers of edible wild plants based in 13 markets at the Yaounde city, between March and April 2012. A total of 304 citations of plants were recorded, using 29 plant species in 32 different food recipes. Fruits and seeds are the most used plant parts, while spices and mouth fruits are in this order the main type of usages observed. Unit prices of sold plants vary from 0.49 FCFA/g to $20 \mathrm{FCFA} / \mathrm{g}$ with an average price per gram of $4.28 \pm 3.1$ FCFA ( 1 USD $=588$ FCFA). Those prices are high compared to other countries and to other cities in Cameroon for the same plant species. Further researches on the monitoring of sellers during a whole year and the gathering of floristic and structural data on food plants are important for establishing their endangerment and thinking of scenarios of sustaining the trade. (C) 2016 International Formulae Group. All rights reserved.
\end{abstract}

Keywords: Ethnobotanical surveys, edible wild plants, recipes, price per gram, Yaoundé. 


\section{INTRODUCTION}

La forêt fournit des biens et services qui sont essentiels pour 1,2 milliards de personnes dans le monde. En Afrique, la survie de 60 à $80 \%$ des populations pauvres dépend directement des ressources naturelles. Les forêts d'Afrique centrale couvrent une superficie d'environ 241 millions d'hectares dont plus de la moitié représente le bassin du Congo considéré comme le second massif forestier tropical après celui de l'Amazonie. Le bassin du Congo abrite la faune et la flore africaines les plus diversifiées et offrent à la multitude des peuples qui y vivent de nombreux produits et services. Les services qu'offre la forêt sont divers : loisirs, tourisme, régulation du climat,...Les produits sont distingués en deux grands groupes à savoir les produits forestiers ligneux constitués essentiellement du bois lorsqu'il est utilisé comme bois d'œuvre (bois d'œuvre industriel et sciage artisanal), et les produits forestiers non ligneux (PFNL). Les produits forestiers non ligneux peuvent être distingués en trois groupes dont les champignons, les produits forestiers non ligneux de nature animale (chasse, chenilles, escargot, ...) et les produits forestiers non ligneux de nature végétale (plantes alimentaires, plantes médicinales et les produits de service).

Lorsque l'on parle de la forêt dans les pays $\mathrm{du}$ bassin du Congo, on pense tout d'abord au bois d'œuvre et dans une certaine mesure au bois-énergie et à la chasse. Les autres produits forestiers non ligneux encore appelés «récoltes cachées » sont négligés et leur contribution à l'économie nationale (produit intérieur brut) est souvent très mal appréciée faute de données statistiques fiables. La mauvaise appréciation observée est ellemême liée à la méconnaissance de ces produits tant sur le plan qualitatif que quantitatif (Awono et al., 2013; Betti, 2007a, b). Pourtant, les «récoltes cachées» ont toujours été et continuent d'être une composante importante de la culture des populations africaines. Ils font non seulement partie des modes de vie «traditionnels » de ces peuples, mais ils demeurent essentiels et indispensables à la qualité de vie de bon nombre de ménages, tant en zone rurale qu'urbaine (Ngonzo et al., 2008). Les récoltes cachées contribuent à la lutte contre la pauvreté, à l'équilibre alimentaire et à la sécurité des populations dans les zones urbaines et rurales (Tchatat et Ndoye, 2006 ; Abalo Atato et al., 2010; Crush et Frayne, 2011; Lauren, 2013) Les marchés locaux jouent un rôle important en permettant aux ménages dépendants des forêts de réaliser une part significative de leurs revenus en vendant des PFNL. L'importance grandissante de la présence des récoltes cachées dans les marchés des villes est liée à l'exode rural massif des populations rurales vers les villes. Ces populations, qui étaient habituées aux aliments forestiers trouvent ici un palliatif à leurs besoins. Elles ont une préférence aux produits forestiers comparés aux produits agricoles comme le riz; mais seulement elles sont limitées par les budgets jugés trop faibles. Lauren (2013) démontre que les populations forestières installées dans des grandes villes au Cameroun ne vont vers les produits agricoles importés que du fait de leur faible prix d'achat comparé aux produits forestiers et notamment aux plantes alimentaires spontanées.

L'ethnobotanique désigne la botanique des sociétés. Botanique ici est prise dans un sens large : analyse descriptives des espèces végétales, écologie, .... L'ethnobotanique étend également son champ de recherche aux applications pratiques de la biologie telles que l'usage des plantes (médecine, rites, alimentation) ainsi qu'aux sciences appliquées. Elle se penche sur les relations possibles entre les espèces et l'organisation sociale et peut-être même pénétrer dans l'univers mythologique et cosmogonique. La méthode ethnobotanique a toujours été considérée comme la voie la plus rapide et sûr dans le processus de valorisation et de gestion durable des ressources forestières (Betti, 
2001). L'approche ethnobotanique permet d'améliorer les connaissances des produits forestiers non ligneux aussi bien sur le plan qualitatif que quantitatif. Sur le plan qualitatif, la recherche ethnobotanique visera, en plus des questions d'identification claire des plantes citées, à caractériser les usages traditionnels ou recettes qui sont faits de ces plantes par les populations locales. Sur le plan quantitatif, la recherche ethnobotanique (ethnobotanique quantitative) visera à préciser les quantités des produits existant et prélevés dans la forêt, les quantités consommées et celles vendues dans les marchés en vue d'appréhender des schémas de valorisation ou de gestion durable de ces produits.

Ce travail d'ethnobotanique analyse les usages traditionnels des plantes alimentaires spontanées vendues dans les marchés de Yaoundé, capitale du Cameroun.

\section{MATERIEL ET METHODES}

\section{Milieu}

Yaoundé est localisée à $3^{\circ} 5^{\prime}$ de latitude Nord et $11^{\circ} 31^{\prime}$ de longitude Est à la lisière de la grande forêt du Sud Cameroun, à $200 \mathrm{~km}$ environ de la côte Atlantique.

La ville de Yaoundé (Région du Centre) appartient au climat équatorial guinéen, sous-type subéquatorial à 4 saisons alternantes : (1) une grande saison sèche qui va de mi-novembre à mi-mars (2) une petite saison de pluie qui se situe entre mi-mars et mi-juin, (3) une petite saison sèche de mi-juin à la fin août et (4) une grande saison de pluie allant de septembre à mi-novembre. La ville de Yaoundé se trouve dans le domaine de la forêt dense humide camerounaise. Dans ce domaine qui couvre une superficie d'environ 20 millions d'hectares, Yaoundé se situe dans la région guinéo-congolaise et plus précisément dans la zone dite de forêts "semicaducifoliées". Ces forêts se distinguent essentiellement des forêts "toujours vertes" du fait que : les grands arbres sont plus nombreux avec des fûts globalement plus rectilignes et des écorces plus uniformément grises, les cimes sont tabulaires et relativement plus rares, le feuillage d'un grand nombre d'espèces arborescentes est caduc, les petits arbustes du sous-bois sont souvent ramifiés et les petits arbustes monocaules sont rares, la strate herbacée est plus développée par suite d'un éclairement du sol en général plus élevé, les lianes sont aussi nombreuses mais dans l'ensemble moins volumineuses, les épiphytes sont moins abondants sur les troncs (Letouzey, 1985 cit. Betti, 2001).

Avec une superficie de $304 \mathrm{~km}^{2}$, la ville de Yaoundé compte environ 2 million d'habitants et constitue la capitale politique du Cameroun. Toutes les tribus y sont représentées, mais l'ethnie autochtone dominante est celle des "Ewondo". D'autres tribus telles que les Eton et les Mbida-mbane sont fixées au voisinage de cette ville (Obala, Mfou, ...). La tribu Ewondo est un rameau de la branche Béti (pluriel de $\mathrm{Nti}=$ seigneur) du groupe PAHOUIN (Fang, Boulou, Béti) (Betti, 2001).

\section{Matériel}

Le matériel utilisé est constitué des fiches d'enquêtes pour les interviews, des sacs en plastique pour la collecte des échantillons, et d'une balance électronique de marque SOEHNLE pour les pesées.

\section{Méthode}

Dans ce travail, les plantes alimentaires désignent aussi bien les plantes dont les produits sont consommés directement et les plantes dont les produits et notamment les feuilles sont utilisées comme emballage dans la confection de certains mets ou la conservation des autres produits alimentaires comme par exemple les feuilles de Maranthaceae, qui servent à l'emballage des «bâtons de manioc ».

La méthode d'enquête utilisée est celle dite « méthode d'enquête ethnobotanique participative ». Cette méthode consiste à interroger les informateurs volontaires sur l'usage traditionnel des plantes utiles. 
L'enquête a été réalisée de mars 2012 à avril 2012 auprès des vendeurs des plantes alimentaires spontanées installés dans les marchés de Yaoundé. Chaque informateur qui acceptait répondre aux questions a été identifié par son nom, le marché, le sexe et son groupe ethnique. Pour chaque organe végétal exposé, le nom vernaculaire de l'espèce a été noté ainsi que l'usage. Celui-ci pouvait être l'amuse-gueule, le légume, le plat principal, l'épice ou condiment ou alors le thé. Les amuse-gueule ou fruits de bouche sont des plantes qui se consomment soit directement, soit après transformation en dehors du repas principal. Les légumes sont des plantes potagères dont les organes (feuilles, fruits, graines, ...) sont utilisés pour la confection des plats d'accompagnement. Le plat principal constitue l'aliment de base du repas des ménages de la zone indiquée. L'épice ou condiment est une substance aromatique qui sert à assaisonner les mets. Pour le thé, l'organe végétal est utilisé en décoction. Les exsudats sont des substances qui sont sécrétées par les plantes elles-mêmes ou extraites pour être utilisées.

Les échantillons de plantes ont été achetés chez chaque vendeur, pesés à l'aide de la balance électronique de marque SOEHNLE et les prix correspondant ont été relevés. Il convient de mentionner que l'idée d'acheter un échantillon de plantes a suscité la motivation des vendeurs à coopérer et participer activement aux enquêtes. Un total de 102 vendeurs de plantes alimentaires spontanées, distribués dans 13 groupes ethniques et 13 marchés a été ainsi interviewé tels qu'indiqué dans le Tableau 1. Les échantillons d'organes végétaux ont été emballés dans des sachets en plastique étiquetés et ramenées à l'Herbier National du Cameroun pour identification. Celle-ci a été facilitée grâce à l'aide du Directeur National de cet herbier et des techniciens locaux. Les données ont été analysées par le nombre de citations, de recettes et par la variation des prix. Chaque fiche d'enquête comportait une information unique appelée «citation» (Betti, 2001, 2002) et qui consistait en neuf éléments: identification du vendeur (nom, sex, ethnie, marché), le nom de la plante, l'organe végétal utilisé, le type d'usage, le poids de l'échantillon et le prix correspondant. Une citation unique d'un seul vendeur ou alors plusieurs citations similaires au niveau de la plante, de l'organe végétal, et du type d'usage fournis par différents vendeurs constituent une recette (Betti et Mebere, 2011).

Toutes les informations collectées ont été analysées par le logiciel Excel 2010. Chaque ligne du tableur Excel correspondait à une citation (enregistrement). Les recettes ont été caractérisées essentiellement par trois éléments : les types d'organes végétaux, les types d'usage, ainsi que les prix du gramme. Le prix du gramme d'une recette donnée est assimilé à la moyenne des prix du gramme des citations liées à cette recette.

\section{RESULTATS}

\section{Profil socio-économique des enquêtés}

Les 102 vendeurs interrogés sont constitués essentiellement des femmes $(88,24 \%)$. Ces vendeurs appartiennent majoritairement aux groupes Bamiléké (48 vendeurs ; $47 \%$ du total) et Eton $(28 ; 27,4 \%)$.

\section{Liste des plantes spontanées alimentaires recensées \\ Un total de 29 espèces végétales} distribuées dans 25 genres et 23 familles ont été recensées. Ces espèces sont présentées dans le Tableau 2 par ordre alphabétique avec leur nombre et pourcentage de citation et de recette. Les familles les plus représentées sont les Zingiberaceae (3 espèces), Arecaceae, Fabaceae, Clusiaceae et Rhamnaceae (2 espèces chacune). Les espèces les plus citées comme plantes alimentaires sont Afrostyrax lepidophyllus $(12,5 \%$ de citations $)$, Aframomum citratum, Ricinodendron heudelotii et Tetrapleura tetraptera avec chacune $7,5 \%$ des citations. 


\section{Caractéristiques des recettes}

Un total de 304 citations faisant ressortir 32 recettes de plantes alimentaires a été recensé dans les marchés. Ces recettes sont représentées dans le Tableau 3 par ordre alphabétique des espèces, des organes végétaux, et des types d'usages. Les recettes les plus citées sont: le condiment obtenu du fruit de Tetrapleura tetraptera $(22,6 \%$ de vendeurs, 10 marchés et 8 groupes ethniques), le condiment obtenu des amandes de Ricinodendron heudelotii (22,6\% de vendeurs, 11 marchés, 9 groupes ethniques), le condiment obtenu des écorces de Afrostyrax lepidophyllus (21,5\% de vendeurs, 10 marchés et 6 groupes ethniques) et les légumes obtenus des feuilles de Gnetum africanum (19,6\% de vendeurs, distribuées dans 9 marchés et 4 groupes ethniques).

Sept types d'organes végétaux ont été cités : les amandes, écorces, racines, feuilles, fleurs, fruits et les graines (Figure 1). Les fruits sont l'organe végétal le plus représenté avec $74 \%$ de citations.

Les plantes alimentaires spontanées vendues dans les marchés de Yaoundé font l'objet de six types d'usages (Figure 2) à savoir: les fruits de bouche, les condiments, les emballages, les ferments, les huiles et les légumes. Les espèces à usages condimentaire sont les plus vendues avec $67,3 \%$ de citations.

\section{Les prix des plantes}

Le Tableau 4 présente le prix moyen du gramme obtenu par organe végétal et par espèce alimentaire dans les marchés. Les prix moyens par gramme varient de $20 \mathrm{FCFA} / \mathrm{g}$ à 1 FCFA/g pour une moyenne de 4,5 3,1 FCFA. La cola (20 FCFA/g), les fleurs de Syzygium aromaticum (12,5 FCFA/g) et les fruits de Piper guneense (12,5 FCFA/g) apparaissent dans cet ordre comme les produits les plus chers dans les marchés. Les graines de palmistes (Elaeis guineensis) et les feuilles de Megaphrynium macrostachyum sont les produits les moins chers car vendus seulement à 1 FCFA/g.



Figure 1 : Organes végétaux vendus comme alimentaires sur les marchés de Yaoundé. 


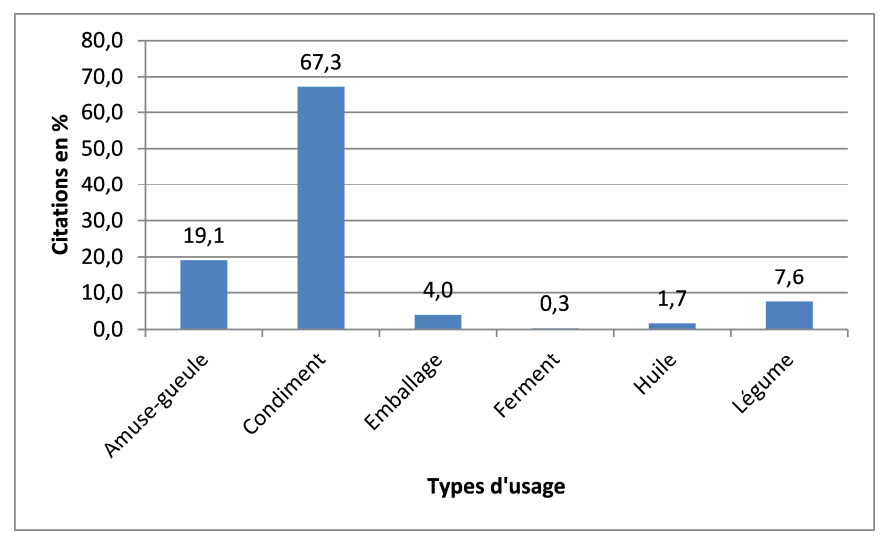

Figure 2: Types d'usages des plantes alimentaires spontanées vendues sur les marchés de Yaoundé.

Tableau 1: Liste des informateurs en fonction des marchés.

\begin{tabular}{|c|c|c|}
\hline Marchés & Codes vendeurs & $\begin{array}{l}\text { Nombre de } \\
\text { vendeur }\end{array}$ \\
\hline $8^{\text {ème }}$ & BmF1, BmF13, BmF30, BmF32, BmF4, MaF1 & 6 \\
\hline Carrière & BmF17, BmF3, BmF31 & 3 \\
\hline Etoudi & $\begin{array}{l}\text { BmF35, BmF38, BmF39, BmF40, BmF45, BmF5, BmF6, SaF1, } \\
\text { SaF3, SaF4, SaF6, SaF8 }\end{array}$ & 12 \\
\hline $\begin{array}{l}\text { Gare } \\
\text { voyageur }\end{array}$ & $\begin{array}{l}\text { BmF12, BmF36, BmF46, BmF7, BmF8, EtF1, EtF25, EtF5, FoF1, } \\
\text { FoF2, FoF3, FoF4, FoF5, SaF2, SaF4, SaF5, SaF7, SaF8 }\end{array}$ & 18 \\
\hline \multirow{2}{*}{ Madagascar } & BeF1, BwF1 & 2 \\
\hline & BmM15 & 1 \\
\hline Melen & BmF29 & 1 \\
\hline Mendong & BmF12, BmF34, BmF43 & 3 \\
\hline Mfoundi & $\begin{array}{l}\text { BmF10, BmF11, BmF2, BmF33, BmF41, BmF42, BmF44, BmF9, } \\
\text { EtF4 }\end{array}$ & 9 \\
\hline \multirow[t]{2}{*}{ Mokolo } & $\begin{array}{l}\text { BkF1, BmF19, BmF20, BmF21, BmF22, BmF23, BmF24, BmF25, } \\
\text { BmF26, BmF27, BmF37, BmF47, BmF48, BmF49, BsF1, BsF5, } \\
\text { BtF1, BtF3, EtF10, EtF13, EtF14, EtF15, EtF16, EtF17, EtF18, } \\
\text { EtF2, EtF23, EtF24, EtF26, EtF28, EtF3, EtF7, EtF8, EtF9, MaF2 }\end{array}$ & 35 \\
\hline & $\begin{array}{l}\text { BfM1, BmM16, BmM18, BtM2, EtM11, EtM12, EtM6, EwM1, } \\
\text { FoM8 }\end{array}$ & 9 \\
\hline \multirow{2}{*}{ Mvogbeti } & BmF28, BsF2, EtF19, EtF20, EtF21, EtF22 & 6 \\
\hline & FoM9, FoM10 & 2 \\
\hline \multirow{2}{*}{ Mvogmbi } & BmF14, BsF4, EtF27, EwF2 & 4 \\
\hline & BsM3 & 1 \\
\hline Total melen & FoF2, FoF6, FoF7 & 3 \\
\hline Yoyo bar & $\mathrm{BdF} 1$ & 1 \\
\hline
\end{tabular}


Tableau 2 : Liste de plantes sauvages alimentaires vendues dans les marchés de Yaoundé.

\begin{tabular}{|c|c|c|c|}
\hline Noms scientifiques & Familles & Nombre de citations & $\%$ citations \\
\hline Adansonia digitata $\mathrm{L}$. & Malvaceae & 4 & 1,3 \\
\hline Aframomum daniellii (Hook. f.) K. Schum. & Zingiberaceae & 19 & 6,3 \\
\hline Aframoтит melegueta K. Schum. & Zingiberaceae & 16 & 5,3 \\
\hline Aframomum citratum & Zingiberaceae & 22 & 7,3 \\
\hline Afrostyrax lepidophyllus Mildbr. & Styracaceae & 38 & 12,5 \\
\hline Balanites aegyptiaca & Balanitaceae & 8 & 2,6 \\
\hline Cola acuminata (P. Beauv.) Schott \& Endl. & Malvaceae & 2 & 0,7 \\
\hline Combretum micranthum G. Don & Combretaceae & 5 & 1,7 \\
\hline Dacryodes edulis (G. Don) H. J. Lam & Burseraceae & 2 & 0,7 \\
\hline Elaeis guineensis Jacq. & Arecaceae & 5 & 1,7 \\
\hline Garcinia kola Heckel & Clusiaceae & 10 & 3,3 \\
\hline Garcinia lucida Vesque & Clusiaceae & 1 & 0,3 \\
\hline Gnetum africanum Welw. & Gnetaceae & 20 & 6,6 \\
\hline Irvingia gabonensis (Aub.-Lec. ex O'R) Bail. & Irvingiaceae & 19 & 6,3 \\
\hline $\begin{array}{l}\text { Megaphrynium macrostachyum (Benth.) Milne- } \\
\text { Redh. }\end{array}$ & Marantaceae & 12 & 4 \\
\hline Mondia whitei (Hook. F.) Skeels & Asclepiadaceae & 7 & 2,3 \\
\hline Monodora myristica (Gaertn.) Duanal & Annonaceae & 10 & 3,3 \\
\hline Phoenix dactylifera & Arecaceae & 5 & 1,7 \\
\hline Piper guineense Schum. \& Thonn. & Piperaceae & 16 & 5,3 \\
\hline Ricinodendron heudelotii P. ex Heck. & Euphorbiaceae & 23 & 7,6 \\
\hline Scorodophloeus zenkeri Harms & Fabaceae & 5 & 1,7 \\
\hline Sesamum indicum $\mathrm{L}$. & Pedaliaceae & 6 & 2 \\
\hline Solanum gilo & Solanaceae & 6 & 2 \\
\hline Syzygium aromaticum & Myrtaceae & 1 & 0,3 \\
\hline Tamarindus indica $\mathrm{L}$. & Fabaceae & 6 & 2 \\
\hline Tetrapleura tetraptera (Schum. \& Thonn.) Taub. & Mimosaceae & 23 & 7,6 \\
\hline Ziziphus jujubaL. & Rhamnaceae & 4 & 1,3 \\
\hline Ziziphus mauritiana Lam. & Rhamnaceae & 3 & 1 \\
\hline Total/moyenne & & 303 & \\
\hline
\end{tabular}


J. L. BETTI et al. / Int. J. Biol. Chem. Sci. 10(4): 1678-1693, 2016

Tableau 3 : Liste des recettes des plantes alimentaires sauvages vendues dans les marchés de Yaoundé.

\begin{tabular}{|c|c|c|c|c|c|c|}
\hline Recettes & Espèces végétales & $\begin{array}{l}\text { Organes } \\
\text { végétaux }\end{array}$ & Utilisations & Codes des informateurs & Vendeurs & $\begin{array}{c}\% \\
\text { Vendeurs }\end{array}$ \\
\hline Rec1 & Adansonia digitata & Fruit & Fruit de bouche & FoF2, FoF5, FoF6, FoF7 & 4 & 3,9 \\
\hline $\operatorname{Rec} 2$ & Aframomum daniellii & Fruit & Condiment & $\begin{array}{l}\text { BdF1, BeF1, BmF1, BmF13, BmF14, BmF2, BmF29, BmF3, } \\
\text { BmF30, BmF31, BmF33, BmF34, BmF35, BmF36, BmF4, } \\
\text { EtF23, EtF9, MaF1, SaF8 }\end{array}$ & 19 & 18,6 \\
\hline $\operatorname{Rec} 3$ & Aframoтит melegueta & Fruit & Condiment & $\begin{array}{l}\text { BdF1, BmF13, BmF29, BmF30, BmF31, BmF34, BmF36, } \\
\text { BmF38, BmF39, BsF1, BsF4, EtF22, EtF25, EtF9, MaF1, SaF3 }\end{array}$ & 16 & 15,7 \\
\hline $\operatorname{Rec} 4$ & Aframomum citratum & Feuille & Condiment & BmF1, BmF13, BmF30, BmF36, BmF4, BmF40, BsF1, SaF4 & 8 & 7,8 \\
\hline $\operatorname{Rec5}$ & Aframomum citratum & Fruit & Condiment & $\begin{array}{l}\text { BdF1, BeF1, BmF17, BmF2, BmF23, BmF3, BmF30, BmF42, } \\
\text { BmF43, BwF1, EtF24, EtF25, EtF26, EtF27 } \\
\text { BdF1, BmF1, BmF13, BmF17, BmF2, BmF24, BmF25, BmF26, }\end{array}$ & 14 & 13,7 \\
\hline Rec6 & Afrostyrax lepidophyllus & Ecorce de tige & Condiment & $\begin{array}{l}\text { BmF29, BmF31, BmF4, BmF41, BmF43, BmF44, BmF45, } \\
\text { BmF46, BmF7, BsF1, EtF27, EtF9, MaF1, SaF2 }\end{array}$ & 22 & 21,6 \\
\hline $\operatorname{Rec} 7$ & Afrostyrax lepidophyllus & Fruit & Condiment & $\begin{array}{l}\text { BdF1, BmF1, BmF13, BmF2, BmF30, BmF31, BmF34, BmF4, } \\
\text { BmF44, BmF45, BmF46, EtF23, EtF9, MaF1, SaF6, SaF7 }\end{array}$ & 16 & 15,7 \\
\hline Rec8 & Balanites aegyptiaca & Feuille & Légume & FoF2, FoF3, FoF6 & 3 & 2,9 \\
\hline $\operatorname{Rec} 9$ & Balanites aegyptiaca & Graine & Fruit de bouche & FoF1, FoF2, FoF5, FoF6, FoF7 & 5 & 4,9 \\
\hline $\operatorname{Rec} 10$ & Cola acuminata & Fruit & Fruit de bouche & BmF9, BmM15 & 2 & 2,0 \\
\hline Rec11 & Combretum micranthum & Fruit & Fruit de bouche & FoF2, FoF3, FoF5, FoF6, FoF7 & 5 & 4,9 \\
\hline $\operatorname{Rec} 12$ & Dacryodes edulis & Fruit & Fruit de bouche & $\mathrm{BmM} 15, \mathrm{EtF} 2$ & 2 & 2,0 \\
\hline $\operatorname{Rec} 13$ & Elaeis guineensis & Graine & Huile & FoF2, FoF4, FoF5, FoF6, FoF7 & 5 & 4,9 \\
\hline Rec14 & Garcinia kola & Graine & Fruit de bouche & $\begin{array}{l}\text { BmF11, BmM16, BmM18, BmF3, BmF9, BsF2, BsM3, BtM2, } \\
\text { FoM8, FoM9 }\end{array}$ & 10 & 9,8 \\
\hline $\operatorname{Rec} 15$ & Garcinia lucida & Ecorce de tige & Ferment & EtM6 & 2 & 2,0 \\
\hline
\end{tabular}


J. L. BETTI et al. / Int. J. Biol. Chem. Sci. 10(4): 1678-1693, 2016

\begin{tabular}{|c|c|c|c|c|c|c|}
\hline Rec16 & Gnetum africanum & Feuille & Légume & $\begin{array}{l}\text { BmF12, BmF17, BmF20, BmF3, BmF5, BmF8, BmF9, BsF5, } \\
\text { EtM11, EtF18, EtF19, EtF20, EtF21, EtF27, EtF28, EtF4, EtF7, } \\
\text { EtF8, MaF1, MaF2 }\end{array}$ & 20 & 19,6 \\
\hline $\operatorname{Rec} 18$ & Irvingia gabonensis & Amande & Condiment & $\begin{array}{l}\text { BkF1, BmF14, BmF17, BmF19, BmF27, BmF3, BmF36, BmF4, } \\
\text { BmF47, BmF48, BmF49, BmF5, BmF9, EtF16, EtF17, EtF27, } \\
\text { EwM1, MaF1, SaF2 }\end{array}$ & 19 & 18,6 \\
\hline Rec19 & $\begin{array}{l}\text { megaphrynium } \\
\text { macrostachyum }\end{array}$ & Feuille & Emballage & $\begin{array}{l}\text { BmF3, BmF4, BsF4, BtF3, EtF10, EtM12, EtF13, EtF14, EtF15, } \\
\text { EwF2, MaF1, SaF2 }\end{array}$ & 12 & 11,8 \\
\hline $\operatorname{Rec} 20$ & Mondia whitei & Racine & Condiment & BmF5, BmF12, BmF13, BmF31, EtF9, MaF1, SaF1 & 7 & 6,9 \\
\hline $\operatorname{Rec} 21$ & Monodora myristica & Fruit & Condiment & $\begin{array}{l}\text { BdF1, BmF1, BmF13, BmF36, BmF4, BmF9, BwF1, EtF1, EtF2, } \\
\text { SaF2 }\end{array}$ & 10 & 9,8 \\
\hline $\operatorname{Rec} 22$ & Phoenix dactylifera & Fruit & Fruit de bouche & FoF2, FoF4, FoF5, FoF6, FoF7 & 5 & 4,9 \\
\hline $\operatorname{Rec} 23$ & Piper guineensis & Fruit & Condiment & $\begin{array}{l}\text { BdF1, BeF1, BmF1, BmF10, BmF13, BmF17, BmF2, BmF30, } \\
\text { BmF32, BmF36, BmF4, BmF6, BmF9, BsF1, EtF2, SaF2 } \\
\text { BdF1, BeF1, BfM1, BkF1, BmF1, BmF10, BmF14, BmF17, }\end{array}$ & 16 & 15,7 \\
\hline $\operatorname{Rec} 24$ & Ricinodendron heudelotii & Amande & Condiment & $\begin{array}{l}\mathrm{BmF} 2, \mathrm{BmF} 22, \mathrm{BmF} 28, \mathrm{BmF} 29, \mathrm{BmF} 3, \mathrm{BmF} 32, \mathrm{BmF} 36, \mathrm{BmF} 6 \\
\mathrm{BsF} 4, \mathrm{BtF} 1, \mathrm{EtF} 2, \mathrm{EtF} 25, \mathrm{EtF} 27, \mathrm{EtF} 9, \mathrm{MaF} 1\end{array}$ & 23 & 22,5 \\
\hline $\operatorname{Rec} 25$ & Scorophloeus zenkeri & Graine & Condiment & BmF14, BmF29, BwF1, EtF9, MaF1 & 5 & 4,9 \\
\hline $\operatorname{Rec} 26$ & Sesamum indicum & Graine & Fruit de bouche & FoF2, FoF3, FoF4, FoF5, FoF6, FoF7 & 6 & 5,9 \\
\hline $\operatorname{Rec} 28$ & Sygygium aromaticum & Fleur & Condiment & $\mathrm{BeF} 1$ & 1 & 1,0 \\
\hline $\operatorname{Rec} 29$ & Tamarindus indica & Fruit & Fruit de bouche & FoF2, FoF3, FoF4, FoF5, FoF6, FoF7 & 6 & 5,9 \\
\hline $\operatorname{Rec} 30$ & Tetrapleura tetraptera & Fruit & Condiment & $\begin{array}{l}\text { BdF1, BeF1, BkF1, BmF1, BmF10, BmF12, BmF13, BmF14, } \\
\text { BmF17, BmF2, BmF21, BmF29, BmF3, BmF32, BmF37, BmF4, } \\
\text { BsF4, BwF1, EtF2, EtF27, EtF3, EtF5, SaF5 }\end{array}$ & 23 & 22,5 \\
\hline $\operatorname{Rec} 31$ & Ziziphus jujuba & Fruit & Fruit de bouche & BmF6, FoF6, FoM10, MaF1 & 4 & 3,9 \\
\hline $\operatorname{Rec} 32$ & Ziziphus mauritiana & Fruit & Fruit de bouche & FoF2, FoF6, FoF7 & 3 & 2,9 \\
\hline
\end{tabular}


Tableau 4 : Prix moyen du gramme des organes de plantes alimentaires exposés dans les marchés de Yaoundé.

\begin{tabular}{|c|c|c|c|c|}
\hline Espèces & $\begin{array}{l}\text { Organe } \\
\text { végétal }\end{array}$ & $\begin{array}{c}\text { Prix moyen du } \\
\text { gramme } \\
\text { (FCFA: } 1 \text { USD } \\
=588 \text { FCFA) }\end{array}$ & $\begin{array}{c}\text { Nombre de } \\
\text { vendeurs }\end{array}$ & $\%$ vendeurs \\
\hline Adansonia digitata & fruit & 2,6 & 4 & 3,9 \\
\hline Aframomum daniellii & fruit & 8,3 & 19 & 18,6 \\
\hline Aframomum melegueta & fruit & 3,7 & 16 & 15,7 \\
\hline \multirow[t]{2}{*}{ Aframomum citratum } & Feuille & 6,6 & 8 & 7,8 \\
\hline & Fruit & 7,2 & 14 & 13,7 \\
\hline \multirow[t]{2}{*}{ Afrostyrax lepidophyllus } & Ecorce & 6,4 & 22 & 21,6 \\
\hline & Fruit & 8,3 & 16 & 15,7 \\
\hline \multirow[t]{2}{*}{ Balanites aegyptiaca } & Feuille & 2,0 & 3 & 2,9 \\
\hline & Graine & 1,7 & 5 & 4,9 \\
\hline Cola acuminata & fruit & 20,0 & 2 & 2 \\
\hline Combretum micranthum & fruit & 2,4 & 5 & 4,9 \\
\hline Dacryodes edulis & fruit & 3,9 & 2 & 2 \\
\hline Elaeis guineensis & graine & 1,0 & 5 & 4,9 \\
\hline Garcinia kola & graine & 4,4 & 10 & 9,8 \\
\hline Garcinia lucida & écorce & 3,3 & 2 & 2 \\
\hline Gnetum africanum & feuille & 2,0 & 20 & 19,6 \\
\hline Imperata cylindrica & graine & 1,6 & 5 & 4,9 \\
\hline Irvingia gabonensis & amande & 3,4 & 19 & 18,6 \\
\hline Megaphrynium macrostachyum & feuille & 1,0 & 12 & 11,8 \\
\hline Mondia whitei & racine & 3,0 & 7 & 6,9 \\
\hline Monodora myristica & fruit & 2,1 & 10 & 9,8 \\
\hline Phoenix dactylifera & fruit & 2,0 & 5 & 4,9 \\
\hline Piper guineensis & fruit & 12,4 & 16 & 15,7 \\
\hline Ricinodendron heudelotii & amande & 4,6 & 23 & 22,5 \\
\hline Scorophloeus zenkeri & graine & 3,0 & 5 & 4,9 \\
\hline Sesamum indicum & graine & 2,0 & 6 & 5,9 \\
\hline Solanum gilo & fruit & 1,8 & 6 & 5,9 \\
\hline Sygygium aromaticum & fleur & 12,5 & 1 & 1 \\
\hline Tamarindus indica & fruit & 2,1 & 6 & 5,9 \\
\hline Tetrapleura tetraptera & fruit & 1,9 & 23 & 22,5 \\
\hline Ziziphus jujuba & fruit & 4,0 & 4 & 3,9 \\
\hline Ziziphus mauritiana & fruit & 1,4 & 5 & 4,9 \\
\hline Total général/moyenne & & $4,5 \pm 3,1$ & 102 & 100,0 \\
\hline
\end{tabular}

\section{DISCUSSION}

Profil socio-économique des enquêtés

Les enquêtes réalisées dans les marchés de Yaoundé ont montré que les femmes étaient majoritaires parmi les personnes impliquées dans la vente des PFNL. Ces résultats corroborent ceux de Tchatat et
Ndoye (2006) qui attestent que le commerce des PFNL est une activité à majorité pratiquée par les femmes. Les activités de production et de commercialisation des PFNL sont en principe partagées entre les hommes et les femmes. Les activités exigeant de gros efforts sont réservées aux hommes tandis que celles 1687 
qui consomment beaucoup de temps sont réservées aux femmes, dont la commercialisation. N'dri et al. (2008) font la même observation dans la région du Fromager en Côte-d'Ivoire. Le ramassage des PFNL (sauf ceux qui nécessitent de grimper) est fait exclusivement par les femmes. La division traditionnelle du travail dans les ménages conduit les femmes à se spécialiser dans la vente de PFNL tandis que les hommes se spécialisent dans la commercialisation du cacao et du café. Toutefois, on relève que les hommes ont rejoint les femmes pour les produits les plus lucratifs. Les quelques hommes enquêtés sont focalisés sur les PFNL dites à haut potentiel économique à savoir Cola acuminata, Garcinia kola, Ricinodendron heudelotii, Dacryodes edulis et Irvingia gabonensis.

Les vendeurs enquêtés sont répartis dans 13 ethnies appartenant à plusieurs régions du Cameroun. Ce résultat montre que le commerce des PFNL est une activité économique multiethnique. Bien que les enquêtes se soient déroulées uniquement dans les marchés de Yaoundé, les vendeurs majoritaires sont les Bamiléké, les Eton et les Foulbé. Les travaux similaires réalisés dans les marchés de Douala ont également révélé la forte représentativité des Bamiléké (71\%) parmi les vendeurs des produits forestiers non ligneux (Dibong et al., 2011). La domination des populations allogènes dans le commerce des PFNL peut s'expliquer par une immigration importante des Bamiléké, Eton et Foulbé vers Yaoundé. En effet, le moteur de la croissance démographique de Yaoundé est l'immigration, en particulier celle des Bamiléké et des Eton qui tendent à supplanter la population locale (les Ewondo). Ceci explique donc pourquoi les Ewondo représentent seulement $1,96 \%$, des vendeurs à Yaoundé.

\section{Caractéristiques des recettes}

Les fruits ou graines et les feuilles dominent parmi les organes végétaux vendus comme aliments sur les marchés de Yaoundé. Ces résultats concordent avec les travaux conduits au Cameroun et notamment dans la région de l'Extrême Nord (Betti et Mebere, 2011 ; Hamawa, 2013) et sur les marchés de Douala (Priso et al., 2011 ; Dibong et al., 2011). Des résultats similaires ont été également obtenus en République Démocratique du Congo et notamment dans les marchés de Kinshassa (Mutambwe Shango, 2010) et Kisangani (Termote et al., 2012). L'importance des fruits ou graines est due à leur valeur nutritive élevée et aussi du fait que les produits dérivés (huile, ...) peuvent mieux se conserver.

Les condiments et les fruits de bouche sont les types d'usages les plus largement cités dans les marchés de Yaoundé. Ces résultats corroborent ceux de Betti et Mebere (2011) dans l'Extrême-Nord Cameroun. Les espèces telles que Irvingia gabonensis, Ricinodendron heudelotii, Piper guineense, Balanites aegyptiaca, Combretum micranthum, Monodora myristica, Tetrapleura tetraptera, Aframomum daniellii, Afrostyrax lepidophyllus, Tamarindus indica, Solanum gilo, Aframomum melegueta, Scorodophloeus zenkeri, Mondia whitei lorsqu'elles sont vendues dans les marchés, le sont le plus souvent comme condiments sous forme séchée, fraîche ou sous forme de pâte. Les fruits de Tamarindus indica fournissent une boisson rafraîchissante et acidulée riche en vitamine $\mathrm{C}$ très consommée en Afrique de l'Ouest (Arbonier, 2000). Balanites aegyptiaca et Ziziphus mauritiana sont listées parmi les fruits sauvages comestibles vendus sur les marchés de Bangui en République Centrafricaine. La pulpe des fruits des deux espèces est consommée fraîche ou sèche. On réduit aussi parfois les fruits de $Z$. mauritiana en farine pour servir à la cuisson des aliments 
(Apema et al., 2010). Les fruits sauvages contiennent les nutriments vitaux (hydrates de carbones, protéines, et les minéraux) et les vitamines essentiels qui sont très importants pour la croissance des enfants qui sont souvent astreints à la malnutrition et aux maladies connexes (Betti et Mebere, 2011; Hamawa, 2013).

L'usage des feuilles et fruits de Adansonia digitata, Balanites aegyptiaca et Tamarindus indica dans l'alimentation a déjà largement été signalé au Cameroun, et notamment dans la région de l'Extrême-Nord (Betti et Mebere, 2011). Les légumes sauvages, tout comme les autres formes de consommation des plantes alimentaires sauvages, jouent un rôle important dans le bien-être des communautés rurales des pays en voie de développement. Ils remplacent facilement les légumes domestiques en période de disette (Asfaw and Tadesse, 2001). Les légumes sauvages constituent des compléments nutritionnels, très indispensable à l'équilibre alimentaire (Somnasang and Moremo-Black, 2000).

\section{Les prix des plantes alimentaires}

La moyenne du prix par gramme des plantes alimentaires spontanées vendues sur les marchés de Yaoundé est de 4,28 $\pm 3,1$ FCFA ( 1 USD $=588$ FCFA).. Ce prix est largement supérieur aux 0,2 FCFA; 0,1 FCFA ; 0,14 FCFA et 0,16 FCFA obtenus respectivement dans les marchés de Bissandérou, Boudorokuy, Soana et Dedouou au Burkina Faso dans la vente des fruitiers sauvages (Alira, 2004). Il est également supérieur aux 2,23 FCFA et 3,24 FCFA obtenus avec les mêmes méthodes pour les plantes médicinales vendues respectivement dans les marchés de Kousséri (Betti et al., 2011a) et Yaoundé (Betti, 2002). En République Démocratique du Congo (Termote et al., 2012), les prix des trois plantes alimentaires spontanées les plus chères dans les marchés de Kisangani se présentent comme suit: Piper guineense (13,41 USD $/ \mathrm{kg}$ $=0,08 \mathrm{FCFA} / \mathrm{g})$, Gnetum africanum $(7,7$ $\mathrm{USD} / \mathrm{kg}=0,04 \mathrm{FCFA} / \mathrm{g}$ ) et Cola acuminata $(3,36 \mathrm{USD} / \mathrm{kg}=0,02 \mathrm{FCFA} / \mathrm{kg})$. On constate que les prix pratiqués en RDC sont très bas, plus de 100 fois plus faibles que ceux trouvés dans les marchés de Yaoundé. La grande différence observée entre Yaoundé et Kisangani peut être liée tout d'abord aux méthodes utilisées pour apprécier les prix des plantes alimentaires dans les deux études. L'étude menée en RDC a couvert pratiquement toutes les saisons de l'année, puisque conduites en quatre phases de deux mois de collecte. L'étude des plantes alimentaires de Yaoundé par contre n'a été conduite qu'en un mois, de Mars à Avril 2012. Les prix des plantes alimentaires vont certainement varier en fonction de leur disponibilité, laquelle est aussi liée à la saison. La différence des prix peut encore être expliquée par le niveau de développement des deux villes. Yaoundé, est la capitale politique du Cameroun et le lieu de convergence de nombreux groupes ethniques, alors que Kisangani est une capitale de province, la province Orientale en République Démocratique du Congo (RDC) pour être plus précis. Même si la province Orientale peut être vaste comparée aux régions du Cameroun, elle reste tout de même moins développée et avec un coût de vie relativement faible comparé à Yaoundé. La dernière explication peut être faite en rapport avec la localisation géographique de ces deux villes. Yaoundé se trouve en zone de forêt guinéenne, semi décidue, avec des forêts bien éloignées alors que Kisangani se trouve au cœur du bassin du Congo, en pleine forêt sempervirente et très riche sur le plan de la phyto diversité. La disponibilité des plantes alimentaires ne poserait pas un gros problème pour les populations de Kisangani comparées à celles de Yaoundé. Les produits qui arrivent 
sur les marchés sont directement cueillis dans les forêts environnantes. Les différences des prix des plantes sauvages alimentaires observées entre Yaoundé et les autres villes du Cameroun peuvent également s'expliquer par les mêmes raisons évoquées plus haut. Yaoundé est la capitale politique du Cameroun et une grande ville, et les plantes vendues dans cette ville sont plus chères que dans d'autres villes.

Les camerounais installés en ville dépensent la majeure part du budget alimentaire aux récoltes cachées; ils ne vont vers les autres types de produits que du fait des prix élevés des produits forestiers (Lauren, 2013). Plusieurs raisons justifient la cherté des produits forestiers trouvés en ville parmi les plus importantes, on relève l'état dégradé des pistes d'évacuation et la para-fiscalité (Lauren, 2013 ; Betti, 2007b). La parafiscalité inclue des prélèvements et fausses taxes perçues par des fonctionnaires véreux (policiers, gendarmes, forestiers, ...), et est surtout à l'origine de nombreux découragements observés chez les vendeurs des produits forestiers non ligneux au Cameroun (Betti, 2002b).

Les prix des plantes peuvent varier en fonction du vendeur, de l'organe et du poids. Mutambwe Schango (2010) explique que les prix des produits forestiers non ligneux dépendent pour beaucoup du rapport de l'offre et de la demande qui, lui-même est influencé par la saisonnalité des produits. Il signale également qu'ils dépendent aussi de la qualité du produit (état de périssabilité), de la dimension ou grosseur du produit ainsi que du coût de transport et de la localité où est vendu le produit. Certaines plantes très largement connues pour leur usage alimentaire ont des prix élevés. Il s'agit de Piper guneense, Afrostyrax lepidophyllus, Aframomum daniellii, Aframomum citratum, Ricinodendron heudelotii, Garcinia kola, Ziziphus jujuba. Certaines de ces espèces à l'instar de Piper guineense et Ricinodendron heudelotii, sont mentionnées comme faisant l'objet d'un commerce régional (Nigéria) ou même international avec l'Europe et les EtatsUnis (Tabuna, 2000 ; Betti, 2007b).

L'importance des produits forestiers non ligneux (PFNL) est bien établie. Ils représentent aux yeux des populations locales la manifestation la plus évidente de la valeur de la forêt et constituent de ce fait un facteur important dans la conservation des ressources de la forêt. En milieu urbain, les revendeurs (bayam selam) des PFNL entretiennent des familles entières grâce à la revente quotidienne des sèves, des épices et aromates, des excitants, des fruits, des feuilles et des écorces d'origine végétale (Awono et al., 2013). Il existe plusieurs autres expressions qui sont utilisées comme des synonymes renvoyant plus ou moins à la même réalité, au rang desquels «produits autres que le bois», «produits naturels», «produits sauvages», «produits forestiers comestibles », etc. Cette diversité terminologique peut conduire à des différences d'interprétations de la contribution de ces produits aussi bien au bien-être des populations qu'aux efforts de conservations des ressources forestières en général. L'analyse des principaux documents de politique gouvernementale en matière de développement agricole, la foresterie et la réduction de la pauvreté au Cameroun révèle l'engagement du gouvernement Camerounais d'inclure les PFNL dans sa stratégie de développement fondé sur la gestion verte de ressources naturelles et la réduction de pauvreté. Cet engagement se manifeste dans le document stratégique de réduction de la pauvreté de 2003 (DSRP), le document stratégique de croissance et d'emploi; le Document Stratégie de Développement rural (REEI) et la Politique forestière nationale (Foundjem-Tita, 2013). Cependant, de nombreuses difficultés entravent encore l'exploitation écologiquement et 
économiquement rentable des produits spéciaux (Betti, 2007a) pour l'Etat et les individus. Le premier écueil réside dans la méconnaissance quasi-totale de la ressource tant sur le plan qualitatif que quantitatif. Ce problème entraîne plusieurs autres tels la généralisation de la taxe de régénération, de nombreux conflits relevés entre les agents forestiers et les revendeurs d'une part, et entre les exploitants des PFNL et ceux de bois d'œuvre d'autre part, la confusion entre les produits agricoles et forestiers (certains produits comme la cola ou la maniguette = Aframomum melegueta débordent le cadre forestier ou sauvage pour intégrer l'espace agricole), les contraintes de commercialisation qui englobent entre autres le manque d'un système d'information sur les marchés, un cadre réglementaire inadéquat, un secteur mal connu du secteur bancaire (risques élevés pour beaucoup), la non standardisation des unités de mesure, la prééminence du secteur informel, le faible prix d'achat aux producteurs, la faible capacité organisationnelle des acteurs (Betti, 2007a, b ; Awono et al., 2013). Les efforts réalisés ces dernières années par le Cameroun sur Prunus africana, une plante médicinale exportée essentiellement en Europe, sont à encourager et à dupliquer sur d'autres PFNL (Betti et al., 2011b).

\section{Conclusion}

L'étude a permis de donner un aperçu sur les plantes sauvages alimentaires vendues dans les marchés de Yaoundé avec un accent particulier sur la caractérisation des usages et des prix. Elle a mis en exergue la diversité des espèces, des usages, et aussi des prix des plantes alimentaires. Ces plantes jouent un rôle important pour les populations rurales installées en ville. La valorisation de ces produits et le développement des outils de commerce durable nécessite de conduire des études approfondies sur les thématiques diverses permettant de mieux connaître ces produits et les ressources liées. La suite logique de ce travail devrait être (1) le suivi d'un groupe de vendeurs des plantes alimentaires dans toute l'année pour mieux apprécier les fluctuations des prix, et (2) aussi la collect les données en vue de caractériser ces espèces sur le plan floristique et structurale. Ces pistes constituent des éléments incontournables pour spéculer sur la vulnérabilité relative de ces plantes et anticiper sur les stratégies de gestion durable.

\section{REMERCIEMENTS}

Nous tenons à remercier tous les vendeurs qui ont accepté de répondre à nos questions. Ce travail a été rendu possible grâce au soutien logistique et financier du projet Forest-savanna sustainability project (JICA/FOSAS) financé par le Gouvernement du Cameroun en collaboration avec la coopération japonaise (JICA).

\section{CONFLIT D'INTERETS}

Les auteurs déclarent qu'ils n'ont aucun conflit d'intérêts.

\section{CONTRIBUTIONS DES AUTEURS}

JLB a conçu la méthodologie, analysé les données et contribué à la rédaction du manuscrit; AES et CMN ont collecté les données, contribué à la rédaction du manuscrit; SDD a contribué à l'analyse des données.

\section{REFERENCES}

Apema R, Mozouloua D, Madiapevo SN. 2010. Inventaire préliminaire des fruits sauvages comestibles vendus sur les marchés de Bangui. In Systématique et conservation des Plantes Africaines, van der Burgt X, van der Maesen J, Onana JM (eds). Royal Botanic Garden: Kew; 313-319. 
Abalo Atato, Wala K, Batawila K, Woegan AY, Akpagana K. 2010. Diversité Des Fruitiers Ligneux Spontanés du Togo. Fruits, vegetable and cereal science and biotechnology. Global Science Book, 4(1):1-9

Asfaw Z, Tadesse M. 2001. Prospects for sustainable use and development of wild food plants In Ethiopia. Economic Botany, 55: 47-62

Awono A, Eba'a Atyi R, Betti J-L, Ngouhouo Poufoun J, Foundjem Tita D, Tieguhong Chupezi J. 2013 Importance économique et sociale des produits forestiers non ligneux de nature végétale au Cameroun. In Etude de l'Importance Economique et Sociale du Secteur Forestier et Faunique au Cameroun, Eba'a Atyi R, Lescuyer G, Ngouhouo Poufoun J, Fouda Moulendè $\mathrm{T}$ (eds). Ministère des Forêts et de la Faune - CIFOR: Yaonudé ; 129-130.

Alira anarila. 2004. Commercialisation des fruits sauvages et de leurs produits dans la boucle du mouhoun : cas des villages de Bissandérou, Boudorokuy, Soana et de la ville de Dedougou: Diplôme d'ingénieur et du développement rural : Sociologie et Economie rurale. Université polytechnique de BoboDioulasso, Burkina Faso, p.83.

Arbonnier. 2000. Arbres, Arbustes et Lianes des Zones Sèches d'Afrique de l'Ouest. CIRAD, MNHM, UICN : Montpellier, France.

Betti JL. 2001. Usages traditionnels et vulnérabilité des plantes médicinales dans la réesrve du Dja et dans les marchés de Yaoundé, Cameroun. Thèse de Doctorat., Université Libre de Bruxelles, Bruxelles, p.229.

Betti JL. 2002. Medicinal plants sold in Yaoundé markets, Cameroon. Afr. Stud. Monogr., 23(2): 47-64.
Betti JL. 2007a. Plan d'action/stratégie pour une meilleure collecte des données statistiques sur les Produits forestiers non ligneux au Cameroun et recommandations pour les pays de la COMIFAC. Projet Renforcement de la sécurité alimentaire en Afrique centrale à travers la gestion et l'utilisation durable des produits forestiers non ligneux, GCP/RAF/398/GER, FAOCOMIFAC - GTZ. P.180.

Betti JL. 2007b. Perspectives d'une fiscalité appropriée promouvant le Commerce et la gestion durable des Produits forestiers non ligneux en Afrique centrale. Projet Renforcement de la sécurité alimentaire en Afrique centrale à travers la gestion et l'utilisation durable des produits forestiers non ligneux, GCP/RAF/398/GER, FAO COMIFAC - GTZ. P.59.

Betti JL, Mebere Yemefa'a SR. 2011. Contribution à la connaissance des produits forestiers non ligneux du parc national de Kalamaloué, Extrême-nord du Cameroun : les plantes alimentaires. Int. J. Biol. Chem. Sci., 5(1): 291 - 303.

Betti JL, Mébéré Yemefa'a SR, Nchembi Tarla F. 2011a. Contribution to the knowledge of non wood forest products of the far north region of Cameroon: Medicinal plants sold in the Kousséri market. Journal of Ecology and the Natural Environment, 3(7): 241-254.

Betti JL, Belinga SJ, Samba D. 2011b. Stock of Prunus africana stems on mount Cameroon. African Journal of Plant Sciences, 5(13): 781-789.

Crush JS, Frayne GB. 2011. Urban food insecurity and the new international food security agenda. Dev. South. Af., 28: $527-544$.

Dibong SD, Mpondo Mpondo E, Ngoye A, Priso R. 2011. Inventory and biodiversity of species edible wild fruits sold in the markets of Douala, Cameroon. International Journal of 
Applied Biology and Pharmaceutical Technology, 2(3): 303-311.

Foundjem-Tita D. 2013. A new institutional economic analysis of policies governing non-timber forest products and agroforestry development in Cameroon. Doctoral Thesis, Ghent University, Belgium.

Hamawa Y. 2013. Wild edible plants used by Guiziga people of far north region of Cameroon. International Journal of Medicinal and Aromatic Plants, 3(2): 136-143.

N'Dri MTK, Gnahoua GM, Kouassi KE, Traoré D. 2008. Plantes alimentaires spontanées de la région du Fromager (Centre-Ouest de la Côte d'Ivoire) : flore, habitats et organes consommés. Sciences et Nature, 5(1): 61-70.

Ngonzo R, Kamga J, Nyako Z. 2008. Etude de marché sur cinq produits forestiers autres que le bois, Programme partenariat coopératif et mutualiste, SOCODEVI

Priso RJ, Nnanga JF, Etame J, Din N, Amougou A. 2011. Les produits forestiers non ligneux: valeur et importance dans quelques marchés de la région du Littoral-Cameroun. Journal of Applied Biosciences, 40: 2715-2726.

Mutambwe Shango, 2010. Revue Nationale sur les Produits Forestiers non Ligneux (PFNL). Cas de la République Démocratique du Congo. Establishment of Forestry Research Network for ACP Countries (FORENET).

Somnasang P, Moreno-Black G. 2000. Knowing, gathering and eating: knowledge and attitudes about wild food in an Isan village in Northeastern Thailand. Journal of Ethnobiology, 20: 197-216.

Tabuna H. 2000. Evaluation des échanges des produits forestiers non-ligneux entre l'Afrique subsaharienne et l'Europe. FAO, Rome et CARPE, USAID.

Tchatat M, Ndoye O. 2006. Étude des produits forestiers non ligneux d'Afrique Centrale : réalités et perspectives. Bois et Forêts des Tropiques $\mathrm{N}^{\circ} 288$ (2). 